\title{
Cuneiform Neurons Activated during Cholinergically Induced Active Sleep in the Cat
}

\author{
Inés Pose, ${ }^{2}$ Sharon Sampogna, ${ }^{1}$ Michael H. Chase,${ }^{1}$ and Francisco R. Morales ${ }^{1,2}$ \\ ${ }^{1}$ Department of Physiology and the Brain Research Institute, University of California at Los Angeles, Los Angeles, \\ California 90095, and 2Departamento de Fisiología, Facultad de Medicina, Montevideo 11800, Uruguay
}

In the present study, we report that the cuneiform (Cun) nucleus, a brainstem structure that before now has not been implicated in sleep processes, exhibits a large number of neurons that express $c$-fos during carbachol-induced active sleep (AS-carbachol). Compared with control (awake) cats, during AS-carbachol, there was a $671 \%$ increase in the number of neurons that expressed $c$-fos in this structure. Within the Cun nucleus, three immunocytochemically distinct populations of neurons were observed. One group consisted of GABAergic neurons, which predominantly did not express $c$-fos during AS-carbachol. Two other different populations expressed $c$-fos during this state. One of the Fos-positive (Fos ${ }^{+}$) populations consisted of a distinct group of nitric oxide synthase (NOS)-
NADPH-diaphorase (NADPH-d)-containing neurons; the neurotransmitter of the other Fos ${ }^{+}$population remains unknown. The Cun nucleus did not contain cholinergic, catecholaminergic, serotonergic, or glycinergic neurons. On the basis of neuronal activation during AS-carbachol, as indicated by c-fos expression, we suggest that the Cun nucleus is involved, in an as yet unknown manner, in the physiological expression of active sleep. The finding of a population of NOS-NADPH-d containing neurons, which were activated during AS-carbachol, suggests that nitrergic modulation of their target cell groups is likely to play a role in active sleep-related physiological processes.

Key words: cuneiform nucleus; REM sleep; brainstem; immunohistochemistry; Fos; nitric oxide
Active sleep (AS) is a complex behavioral state that is comprised of diverse physiological processes (Steriade and McCarley, 1990; Jones, 1991) (for review, see Siegel, 1994; Rye 1997). Among the distinguishing physiological characteristics of this state are muscular atonia, EEG desynchronization, ponto-geniculo-occipital (PGO) waves, and rapid eye movements.

There is evidence that many of these processes are initiated and/or regulated by brainstem structures. The activity of cholinergic neurons in the laterodorsal tegmental (LDT) and pedunculo-pontine tegmental (PPT) nuclei appears to be critical for the generation of AS (Steriade and McCarley, 1990; Jones, 1991; Hobson, 1992; Rye, 1997). For example, the injection of cholinergic agonists into cholinoceptive structures in the rostral pontine tegmentum triggers a state that is similar in many aspects to naturally occurring AS (George et al., 1964; Baghdoyan et al., 1987, 1989; Morales et al., 1987; Vanni-Mercier et al., 1989). This cholinergically induced AS-like state (AS-carbachol) has also been used to explore the neurophysiological basis of AS and to identify neuronal populations that are involved in the generation and maintenance of this state (Baghdoyan et al., 1989; Shiromani et al., 1992; Shuman et al., 1995; Xi et al., 1997; Capece et al., 1998; Morales et al., 1999).

The expression of the proto-oncogene $c$-fos, detected by the immunocytochemical visualization of its protein product Fos, has been used as a marker of neuronal activity (for review, see Herrera and Robertson, 1996; Chaudhuri, 1997). Since the development of these techniques, we (Yamuy et al., 1993, 1995,

Received Nov. 29, 1999; revised Feb. 10, 2000; accepted Feb. 17, 2000.

This work was supported by United States Public Health Service Grants NS 23426, NS09999, and MH 43362. We thank Gerardo Morales for his technical assistance.

Correspondence should be addressed to Dr. Francisco Morales, Department of Physiology, University of California at Los Angeles School of Medicine, Los Angeles, CA 90095. E-mail: fmorales@ucla.edu.

Copyright (C) 2000 Society for Neuroscience 0270-6474/00/203319-09\$15.00/0
1998; Morales et al., 1999) and others have used the expression of c-fos to identify populations of neurons that are activated during AS (Merchant-Nancy et al., 1992; Shiromani et al., 1992; Maloney et al., 1999).

During the course of anatomical studies of the brainstem, we consistently observed that larger number of neurons within the cuneiform (Cun) nucleus expressed c-fos during AS-carbachol than during wakefulness. This finding is important, because the Cun nucleus has not been implicated previously in any of the physiological processes that occur during AS (for review, see Sakai, 1988; Steriade and McCarley, 1990; Jones, 1991; Hobson, 1992; Siegel, 1994; Rye, 1997).

In the present work, we describe the pattern of $c$-fos expression in this brainstem nucleus. The present results confirm our preliminary observations and provide new evidence of the neurotransmitter phenotype of cells within this structure. A novel subpopulation of noncholinergic Cun neurons that contain nitric oxide synthase (NOS) was found. Because these cells exhibited robust $c$-fos expression during AS-carbachol, we suggest that they participate in the nitrinergic modulation of their target neurons during this state.

\section{MATERIALS AND METHODS}

Eleven adult cats, weighing between 3.0 and $3.5 \mathrm{~kg}$, were used in the present study. Five animals were studied after prolonged periods of AS-carbachol (experimental animals); six cats were kept awake for control periods (control animals).

\section{Surgical procedures}

All experimental procedures were conducted in accordance with the Guide for the Care and Use of Laboratory Animals (Ed 7) and approved by the Chancellor's Animal Research Committee of the University of California at Los Angeles for the protection of research subjects. The surgical procedures to prepare "chronic" cats for state recordings and the delivery of substances within the brainstem have been described previ- 
ously (Morales et al., 1999). Two weeks after surgery, the cats were adapted to a head-restraining device until they exhibited episodes of naturally occurring sleep.

\section{Experimental procedures}

Experimental animals. During the sleep studies, the EEG, EMG, and electro-oculographic activity were recorded. After a baseline recording period of $1 \mathrm{hr}$, the cannula of a $1 \mu \mathrm{l}$ Hamilton syringe that was filled with a solution of carbachol ( $88 \mathrm{~mm}, 16 \mu \mathrm{g}$ in $1 \mu \mathrm{l}$ of saline) was lowered through an access hole in the occipital bone to inject carbachol at the following stereotaxic coordinates: posterior 3.0, lateral 1.3, vertical -3.5 (Berman, 1968). Five cats were injected with $0.1 \mu \mathrm{l}$ of this solution. After a $2 \mathrm{hr}$ recording session, the animals were killed with an overdose of sodium pentobarbital $(60 \mathrm{mg} / \mathrm{kg}$, i.p.).

Control animals. In previous work (Morales et al., 1999), we compared data from AS-carbachol cats with those from animals in quiet wakefulness. In the present report, we examined, as before, two control cats in which the same procedures were followed, except that $0.1 \mu \mathrm{l}$ of saline was injected instead of carbachol. Four additional control animals, which were awake, were included in the present study. Two of these animals were maintained in quiet wakefulness by gentle stimulation of the skin when the cats fell asleep. One control animal was continuously aroused by tactile stimulation of the face and whiskers. The remaining control cat was brought from the vivarium into a laboratory room in which it was allowed to run freely for $2 \mathrm{hr}$. It spent the free time in a state of arousal, during which time it explored the environment. It was then anesthetized and killed. Because there was no statistically significant difference in the number of Fos-positive $\left(\mathrm{Fos}^{+}\right)$cells in the Cun nucleus between the different groups of control animals, the data from these cats were pooled to compare them with data from AS-carbachol animals.

While deeply anesthetized with sodium pentobarbital, all animals were perfused transcardially with 11 of saline, followed by 2.51 of a solution of $4 \%$ paraformaldehyde, $15 \%$ saturated picric acid, and $0.25 \%$ glutaraldehyde in $0.1 \mathrm{M}$ phosphate buffer at $\mathrm{pH}$ 7.4. The brainstem was removed and immersed for a $24 \mathrm{hr}$ post-fixation period in a solution consisting of $2 \%$ paraformaldehyde and $15 \%$ saturated picric acid in 0.1 $\mathrm{M}$ phosphate buffer at $\mathrm{pH}$ 7.4. After post-fixation, the tissue was kept in a solution of sucrose $(30 \%)$ in $0.1 \mathrm{~m}$ phosphate buffer at $\mathrm{pH} 7.4$.

Forty-eight to $72 \mathrm{hr}$ later, the brainstem was frozen and cut into $15-\mu \mathrm{m}$-thick sections using a Reichert-Jung cryostat. As each section was cut, it was placed in one well of a 36 well tray containing a buffered solution [0.1 M PBS containing $0.3 \%$ Triton X-100 and $0.1 \%$ sodium azide (PBST-azide); note that Triton was omitted in those wells assigned for GABA immunocytochemistry]. The first section obtained was placed in well 1 of the set, and consecutive sections were placed in consecutive wells in serial order. Section 37 was placed in well 1, and the procedure was repeated until the entire brainstem was sectioned. Using this method, each well contained a sample of the entire brainstem wherein each section corresponded to a cut $540 \mu \mathrm{m}$ apart from the succeeding section $(15 \times 36)$. The sections contained in different wells were then processed using different antibodies.

\section{Fos immunocytochemistry}

Sections were processed for Fos immunostaining using a polyclonal rabbit antibody (Fos Ab5; Oncogene Research Products, Calbiochem, La Jolla, CA). The free-floating sections were incubated overnight in this antiserum at a dilution of 1:20,000 in PBST-azide. The sections were then rinsed in PBST for $30 \mathrm{~min}$ and incubated for $90 \mathrm{~min}$ in biotinylated donkey anti-rabbit $\operatorname{IgG}$ diluted 1:300 containing 1.5\% normal donkey serum. After rinsing for $30 \mathrm{~min}$, the sections were incubated for $90 \mathrm{~min}$ in ABC complex (Vector Laboratories, Burlingame, CA) at a dilution of 1:200. Peroxidase activity was visualized by reacting the sections with $0.02 \%$ diaminobenzidine tetrahydrochloride and 0.015 hydrogen peroxide in $50 \mathrm{ml}$ of $50 \mathrm{~mm}$ Tris-buffered saline, $\mathrm{pH} 7.5$, for $15-30 \mathrm{~min}$.

\section{Neurotransmitter phenotype}

Sections were treated for labeling of one of the following transmitters or transmitter-related enzymes: choline acetyltransferase (ChAT), tyrosine hydroxilase $(\mathrm{TH})$, glutamate, GABA, glutamic acid decarboxylase (GAD), glycine, serotonin (5-HT), and NOS. NADPH-diaphorase (NADPH-d) chemical activity was examined using nitro blue tetrazolium (NBT).

For ChAT, TH, 5-HT, GAD, and NOS immunocytochemistry, freefloating sections were incubated overnight in one of the following: polyclonal antiserum directed against ChAT (dilution 1:2000; Chemicon,
Temeluca, CA), TH (dilution 1:5000; Pel-Freeze Biologicals, Rogers, AR), 5-HT (dilution 1:10000; Incstar), GAD (dilution 1:6000; Chemicon), or NOS (dilution: 1:500; neuronal isoform; Accurate Chemicals, Westbury, NY). After rinsing in PBST, the tissue was incubated for 90 min in their respective secondary antibody at a dilution of 1:300 for ChAT and TH, 1:1000 for 5-HT, 1:200 for GAD, and 1:500 for NOS. The sections were then rinsed in PBST and treated with the ABC complex (Vector Laboratories standard Elite kit). Peroxidase activity was visualized by reacting the sections with $0.02 \%$ diaminobenzidine tetrahydrochloride (Sigma, St. Louis, MO) and 0.015 hydrogen peroxide in $50 \mathrm{ml}$ of $50 \mathrm{~mm}$ Tris-buffered saline, $\mathrm{pH} 7.6$ for $15-30 \mathrm{~min}$.

For GABA immunoreactivity, an antibody to GABA conjugated to keyhole limpet hemocyanin with glutaraldehyde (dilution 1:3500; ProtosBiotech Corp., New York, NY) was used. Similar procedures as those described above were followed afterwards. The dilution of the biotinylated donkey anti-guinea pig secondary antibody was 1:300 and that of the ABC complex was 1:200.

For glutamate immunoreactivity, a mouse monoclonal antibody to glutamate conjugated to keyhole limpet homocystein with glutaraldehyde (dilution 1:500; Diasorin, Stillwater, MN) was used. The dilution of the biotinylated donkey anti-mouse secondary antibody was 1:200 and that of the ABC complex was 1:200.

For glycine immunoreactivity, a rabbit polyclonal antibody against glycine-conjugated t-thyroglobulin with glutaraldehyde (1:500; Chemicon) were used. The dilution of the biotinylated donkey anti-mouse secondary antibody was 1:200 and that of the ABC complex was 1:200.

To detect NADPH-d chemical activity, sections were incubated in a solution of $0.1 \mathrm{~m}$ PBS, $\mathrm{pH} 7.4,0.3 \%$ Triton X-100, $0.1 \mathrm{mg} / \mathrm{ml} \mathrm{NBT}$, and $1.0 \mathrm{mg} / \mathrm{ml} \beta$-NADPH for 30-60 min. After fixation with a paraformaldehyde solution, the enzyme NADPH-d that remains active is a NOS coenzyme. In selected sections, ChAT immunochemistry was combined with the NADPH-d reactions (for example, see Fig. 8).

After immunocytochemistry, selected sections were counterstained by Pyronin Y.

\section{Data analysis}

Histological sections were examined using an Olympus BX60 microscope (Olympus Optical, Tokyo, Japan). Photomicrographs for neuronal counting and illustrations were obtained by means of a digital camera attached to the microscope and connected to a microcomputer with Photoshop software (Adobe Systems, San Jose, CA). To determine soma size, a $100 \times$ oil immersion objective lens was used. Stained neurons in which the nucleolus was apparent were photographed, and their major and minor soma diameters were measured as described previously (Morales et al., 1999). The value for cell diameter in the present work is that of the sum of the major and minor diameters divided by 2 .

Preliminary observations indicated a considerable number of Fosimmunoreactive cells in the Cun of AS-carbachol cats. Therefore, sections selected for analysis were located between posterior -0.9 and -2.1 encompassing this nucleus in its entirety (Berman, 1968). Ten nonadjacent sections of the brainstem of each cat were used to count Fos ${ }^{+}$nuclei. The mean number of $\mathrm{Fos}^{+}$cells in the Cun nucleus per section and per cat was calculated. The means for AS-carbachol and control animals were compared using the Student's $t$ test. The following formula was used to express the percent increase in number of neurons during AS-carbachol:

$$
\frac{A S-C}{C} * 100
$$

where $A S$ and $C$ are mean number of $\mathrm{Fos}^{+}$cells in AS-carbachol and control, respectively.

\section{RESULTS}

After carbachol microinjection, the experimental cats exhibited a prolonged (1 hr $52 \mathrm{~min} \pm 5 \mathrm{~min})$ active sleep-like state (AS-carbachol).

Figure $1 A$ illustrates the pontomesencephalic region, which is the focus of the present report. The boundaries of the Cun nucleus are the periaqueductal gray medially, the inferior colliculus (IC) dorsally, and the lateral lemniscus laterally. Ventrally, in its rostral portion, the Cun nucleus is separated from the brachium conjunctivum by the lateral portion of the PPT and, in its caudal portion, by the lateral parabrachial subnuclei (Fulwiler and Saper, 1984; Rye et al., 1987; Steriade and McCarley, 1990). 

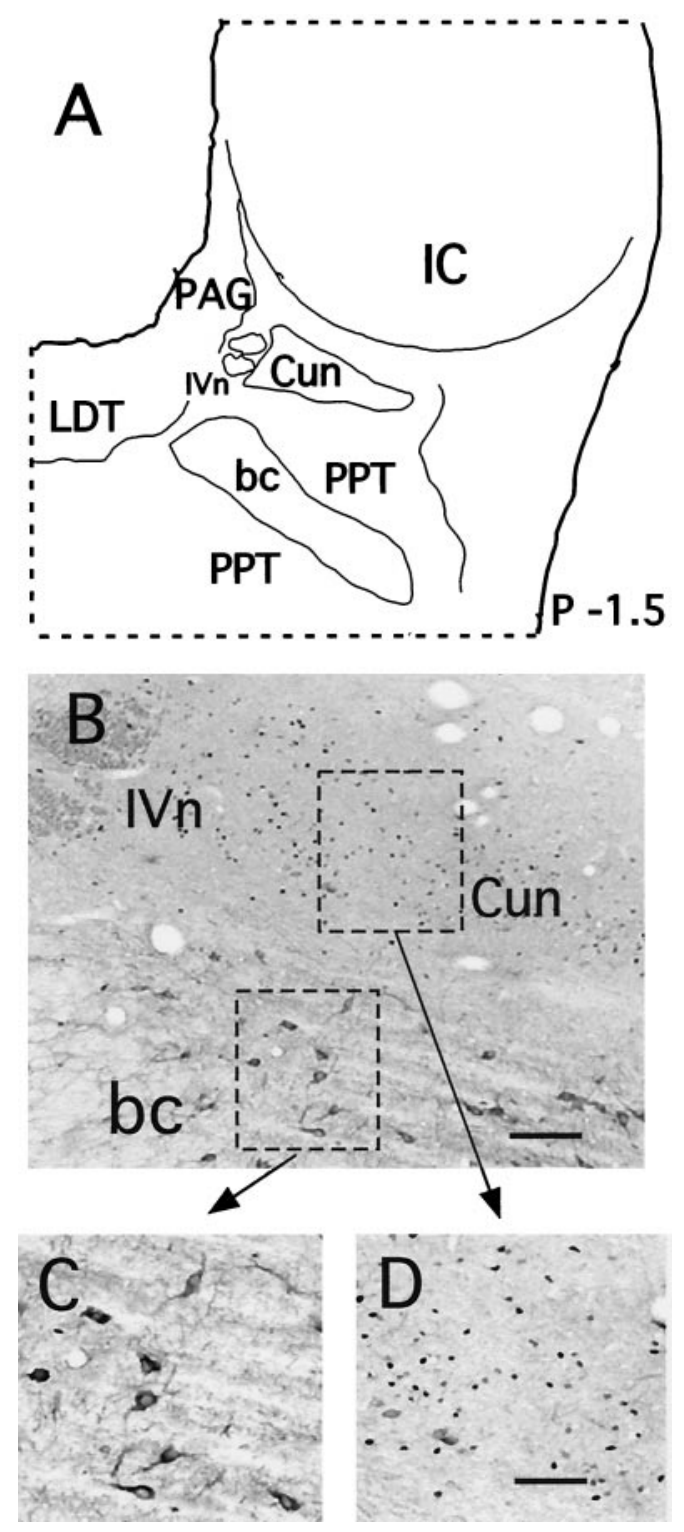

Figure 1. c-fos expression in neurons of the Cun nucleus in an experimental cat. $A$, Diagram of a brainstem section of an experimental animal to show the location of the Cun nucleus and surrounding structures. $B$, The photomicrograph was taken at low magnification $(10 \times)$ to illustrate the concentration of $\mathrm{Fos}^{+}$nuclei in this nucleus. The histological section in the photomicrograph was processed for both ChAT and Fos immunostaining. Neurons expressing ChAT are those dorsal to the brachium conjunctivum; Fos-labeled nuclei are located dorsal to this population of cells. $c$-fos-expressing cells were not ChAT-labeled neurons, and ChATlabeled neurons did not express $c$-fos. The fibers of the IV cranial nerve were transversely cut and can be observed in the top left corner of the photomicrograph. $C$, Higher magnification photomicrograph of the ChAT-labeled cells. $D$, Higher magnification photomicrograph of the Fos $^{+}$cells. Scale bars: $B, 100 \mu \mathrm{m} ; C, D, 60 \mu \mathrm{m}$. $b c$, Brachium conjunctivum; $P A G$, periaqueductal gray; $I V n$, trochlear nerve.

The photomicrograph in Figure $1 B$ was taken from a brainstem section from an experimental cat. The tissue was processed for both ChAT and Fos immunostaining. Selected areas of this section (dashed rectangles) are illustrated at higher magnification in $C$ and $D$. ChAT immunoreactivity appears as a cytoplasmic stain, and Fos immunoreactivity appears as a dark nuclear stain. Numerous $\mathrm{Fos}^{+}$nuclei can be observed ventral to the IC and lateral to the fibers of the trochlear nerve (Fig. 1B). A group of ChAT-

\section{AS-carbachol}
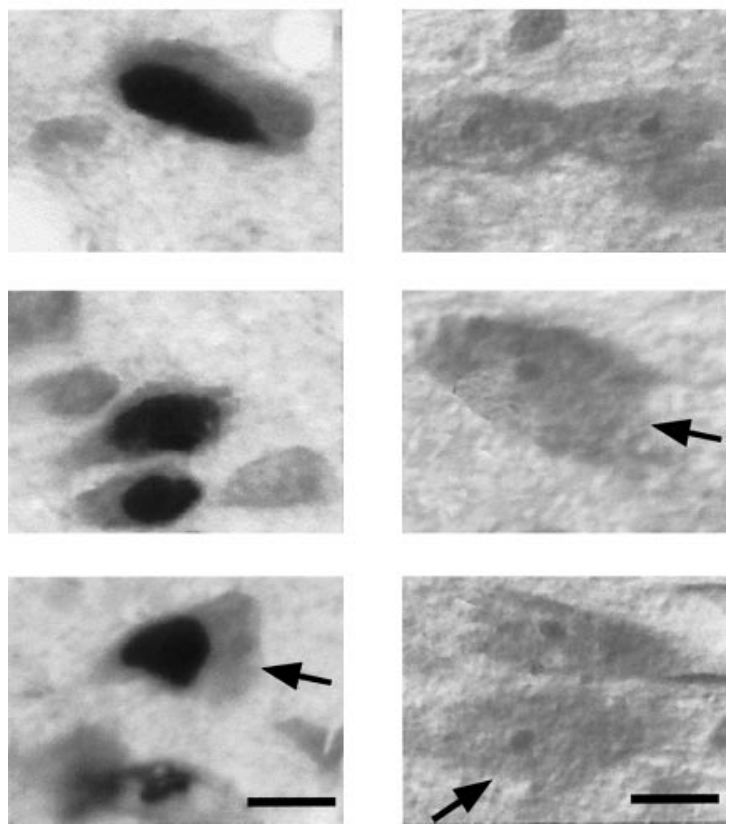

Figure 2. Examples of Cun nucleus neurons illustrating the morphology of their cell bodies. Photomicrographs in the left column illustrate neurons from a section of an experimental animal that was processed for Fos immunostaining and counterstained by Pyronin Y. Right column, as left column, but with tissue obtained from a control animal. The arrows point to triangular cell bodies. The rest of the cell bodies exhibit a fusiform shape. Note that the cytoplasm surrounding the nucleus of the neurons is very thin. Scale bars, $5 \mu \mathrm{m}$.

positive cells lies between the $\mathrm{Fos}^{+}$population and the brachium conjunctivum. A photomicrograph of these cells is presented at higher magnification in Figure $1 C$. This cholinergic population corresponds to the lateral part of the PPT (Jones and Beaudet, 1987; Rye et al., 1987; Vincent and Reiner, 1987). The nuclei of these cholinergic cells, which are not part of the Cun nucleus, did not contain detectable amounts of the Fos protein. Maloney et al. (1999) have described a $22 \%$ increase in the number of Fos ${ }^{+}$ cholinergic neurons of the LDT-PPT under conditions of AS recovery in rats. However, within the lateral PPT, which encompasses the cholinergic cells illustrated in the diagram and in the photomicrograph in Figure 1, these authors did not describe a significant difference in $\mathrm{Fos}^{+}$cholinergic neurons, which is in agreement with our present results.

The photomicrographs in Figure 2 were taken at high magnification from tissue immunostained for the Fos protein and counterstained to reveal the morphology of neuronal cell bodies. The column on the left is from an experimental cat and depicts Fos ${ }^{+}$ neurons. The column on the right is from a control cat. Cun neurons were found to be small (mean soma diameter of $14 \pm 1.6$ $\mu \mathrm{m}$ ), fusiform or polygonal in shape, with a relatively large nucleus and a single nucleolus. The arrows indicate neurons with a triangular profile. With respect to their morphology, the Cun neurons observed in our study were similar to those described by Gioia and Bianchi (1987) in the cat and Rye et al. (1987) in the rat.

In AS-carbachol cats, Cun neurons expressing $c$-fos were observed along the rostrocaudal extent of this structure (Fig. 3). Figure 4 summarizes our results. The number of $\mathrm{Fos}^{+}$cells per 


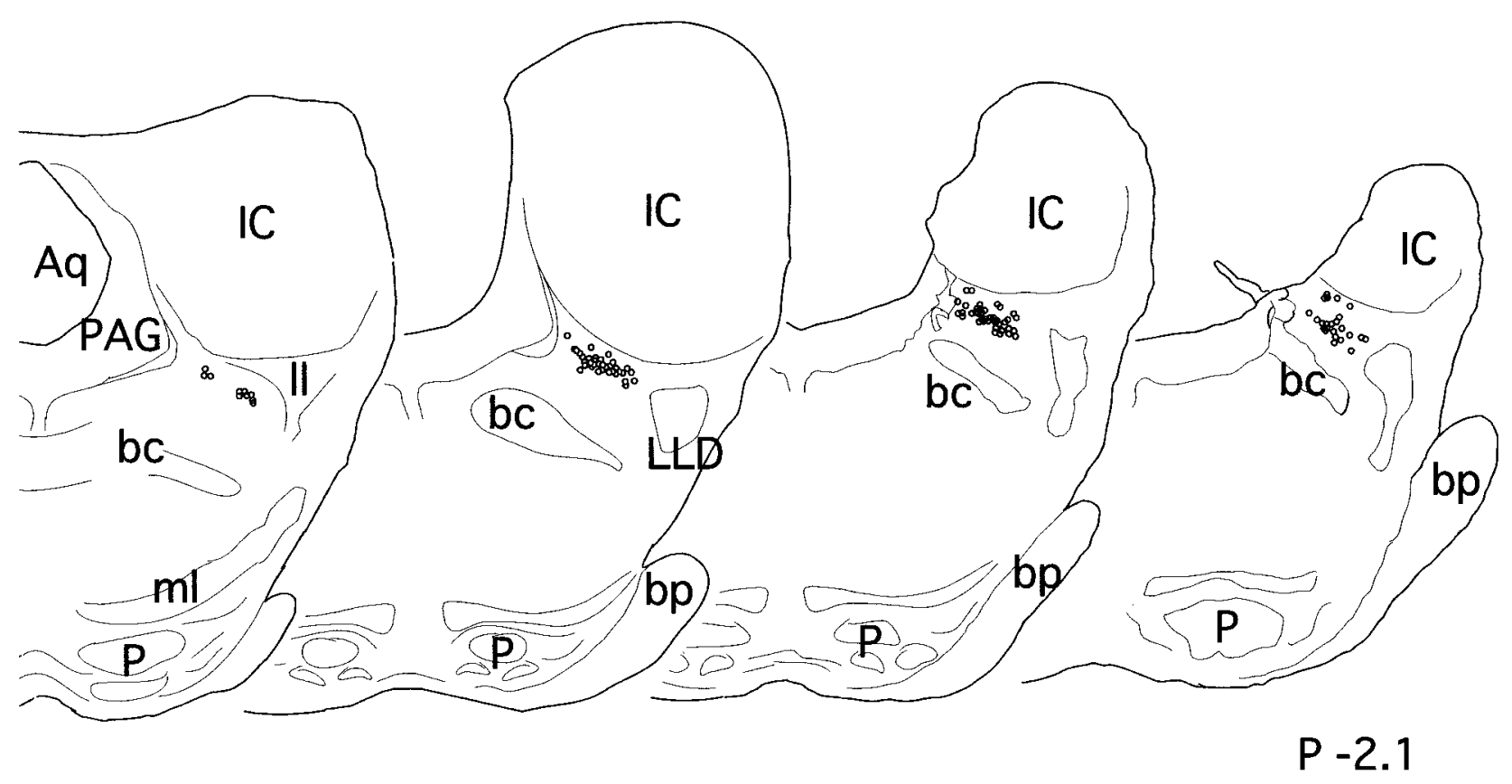

Figure 3. Localization of Fos-labeled neurons of the Cun nucleus at four levels of the cat brainstem. The schematics are from an experimental animal and correspond to coronal sections of the brainstem taken at $540 \mu \mathrm{m}$ intervals. The most posterior section corresponds approximately to posterior -2.1 (Berman, 1968). The circles represent Fos ${ }^{+}$nuclei; tissue was obtained immediately after a long episode of AS-carbachol and processed for Fos protein immunostaining and counterstained by Pyronin Y. $A q$, Aqueduct; $b c$, brachium conjunctivum; $b p$, brachium pontis; $L L D$, dorsal nucleus of the lateral lemniscus; $m l$, medial lemniscus; $P$, pyramidal tract; $P A G$, periaqueductal gray; $l l$, lateral lemniscus.

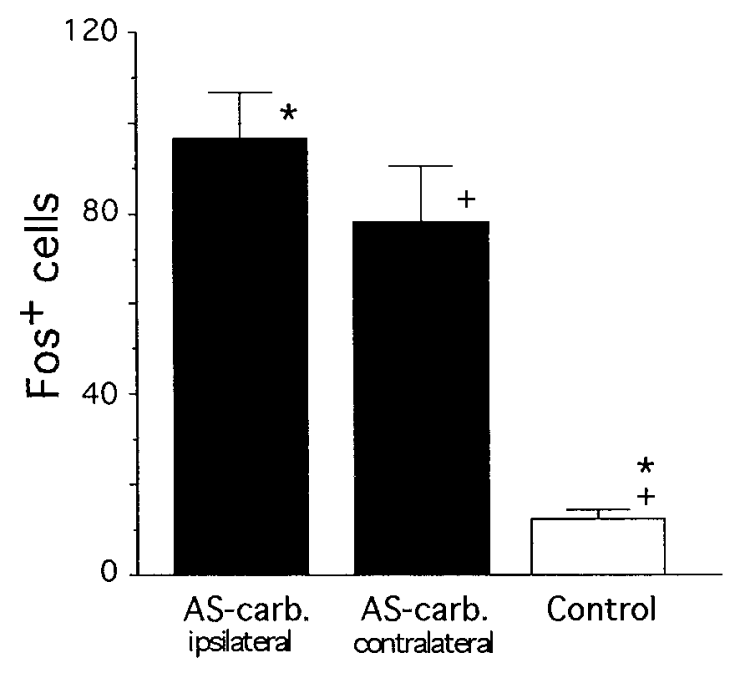

Figure 4. c-fos expression in control and experimental cats. The bar chart represents the mean of the average number of $\mathrm{Fos}^{+}$neurons per section per cat within the Cun nucleus. These data were obtained from five experimental and six control cats. Mean \pm SEM values: control, $12.5 \pm$ 2.0; AS-carbachol, ipsilateral, $96.4 \pm 10.5 ;$ AS-carbachol, contralateral, $78.2 \pm 12.3\left({ }^{*}\right.$ and,$\left.+ p<0.0001\right)$.

section in AS-carbachol cats was $96.4 \pm 10.5$ (mean \pm SEM) on the ipsilateral side and $78.2 \pm 12.3$ on the contralateral side; in control cats, it was $12.5 \pm 2.0$. There was a $671 \%$ increase in the number of $c$-fos-expressing cells on the ipsilateral side and a $523 \%$ increase on the contralateral side in AS-carbachol cats when compared with control animals. In both cases, the differences were statistically significant $(p<0.0001)$. The absolute mean value of $\mathrm{Fos}^{+}$cells in AS-carbachol cats was larger ipsilaterally than contralaterally to the injection side. However, the $p$ value, although low $(<0.09)$, did not reach statistical significance. In control cats, there were no statistical differences in the number of $\mathrm{Fos}^{+}$cells between sides of the brainstem.

\section{Neurotransmitter phenotype}

Figures 5-8 are examples of experiments in which Fos immunocytochemistry was combined with different techniques to label glutamatergic, GABAergic, cholinergic, or NOS-NADPH-d containing neurons.

As shown in the example presented in Figure $5 A, \mathrm{Fos}^{+}$cells in the Cun nucleus did not show glutamate-like immunoreactivity. Glutamate-like reactivity was, however, observed in structures contained in the same or adjacent brainstem sections, for example in the IC or in the mesencephalic trigeminal (Mes-V) nucleus as shown in Figure 5, $B$ and $C$. The observation that neurons in the Cun nucleus did not display glutamate immunoreactivity was unexpected. This issue will be addressed in Discussion. In addition, these neurons did not exhibit serotonergic, catecholaminergic, or glycinergic immunoreactivity.

GABAergic neurons were identified by using either an antibody directed at a GABA-conjugated protein or one directed at GAD. In Figure 6 are examples of these GABAergic neurons in the Cun nucleus. The diagram in Figure $6 A$ illustrates the distribution of these cells [open circles are $\mathrm{GABA}^{+}$, Fos-negative (Fos ${ }^{-}$) neurons; dotted circles are $\mathrm{GABA}^{+}, \mathrm{Fos}^{+}$neurons; filled circles are $\mathrm{Fos}^{+}, \mathrm{GABA}^{-}$cells]. GABAergic cells had a tendency to cluster close to the ventral border of the IC. The great majority $(92 \%)$ of GABAergic cells did not express $c$-fos. The fact that the Cun nucleus contains GABAergic cells is in agreement with the observations of others (Mugnaini and Oertel, 1985; Appell and Behan, 1990).

The results obtained with NADPH-d histochemistry and/or with NOS, ChAT, and Fos immunostaining are shown in Figures 7 and 8. Within the Cun nucleus, there was a population of 

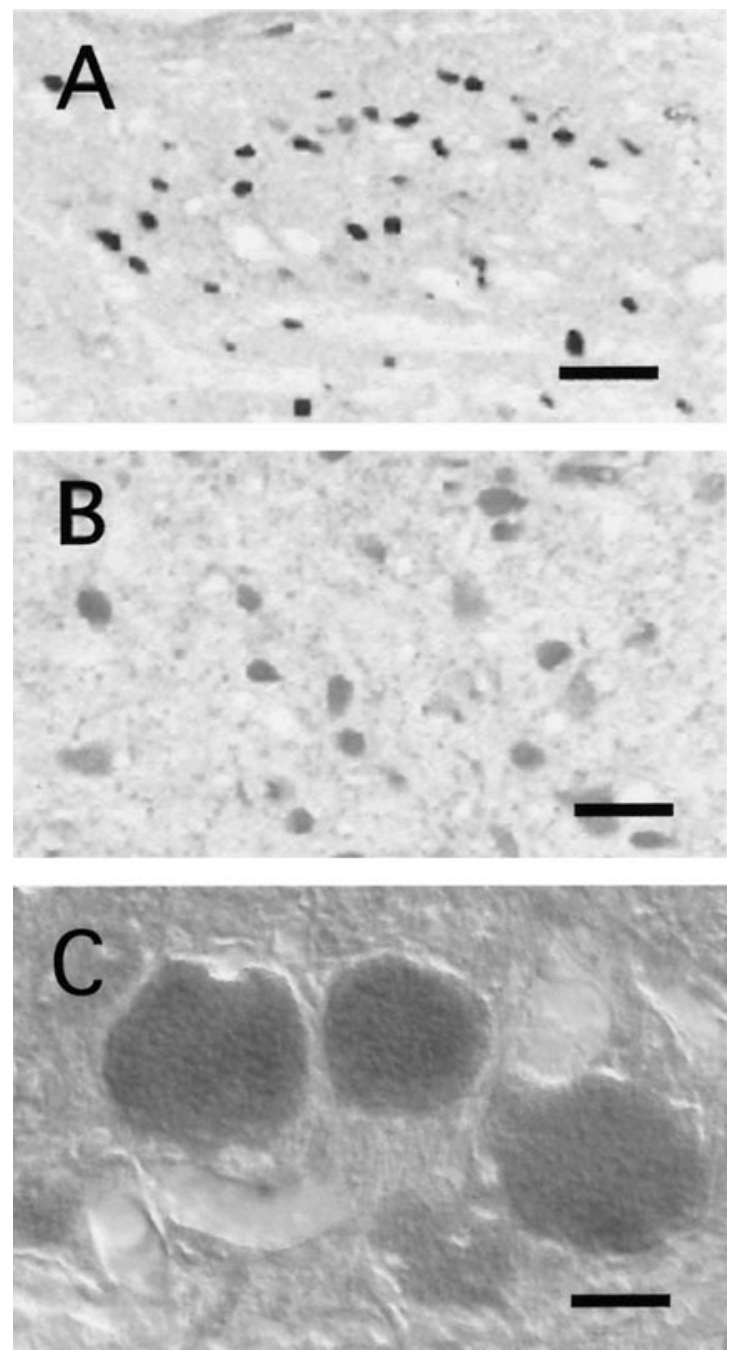

Figure 5. Fos $^{+}$Cun nucleus neurons did not exhibit glutamate-like immunoreactivity. The histological sections in the photomicrographs were processed for both glutamate and Fos immunostaining. $A$ is a photomicrograph of the Cun nucleus, $B$ is from the inferior colliculus, and $C$ is from mesencephalic trigeminal neurons. Scale bars: $A, 50 \mu \mathrm{m} ; B, C$, $25 \mu \mathrm{m}$.

NADPH-d-containing neurons. The photomicrographs in Figure 7 are two examples of these Cun neurons and an example of a NADPH-d positive neuron that belongs to the cholinergic population of the pontine tegmentum. Note the marked morphological differences between these two types of cells.

During AS-carbachol, $50 \%$ of the NADPH-d-containing Cun neurons expressed $c$-fos compared with only $1 \%$ in control, awake cats. The shaded area in Figure $8 A$ indicates the location of these neurons within the central portion of the Cun nucleus. This population was concentrated in the caudal portion of this nucleus, at approximately posterior 2.1 (Berman, 1968), the level of the crossing fibers of the IV nerve (arrow). The photomicrograph in $B$ is a low-magnification $(10 \times)$ presentation of a section processed with NBT to determine NADPH-d activity. Although it is not evident at this magnification, this section was also treated previously to detect $c$-fos expression. NADPH-d activity was visualized as a blue cytoplasmic stain. In this section, both the NADPH-d reactive population in the Cun nucleus and that in the LDT are visible. A view at higher magnification of each of these two regions is illustrated in $C$ and $D$, respectively.
Numerous double-labeled Fos ${ }^{+}$, NADPH-d reactive cells may be observed in Figure 8C. These cells were small (mean soma diameter of $14.5 \pm 1.7 \mu \mathrm{m}$ ), and most of them had a fusiform shape (see $C^{\prime}$ inset and $H$ ). For comparison, the typical large (mean soma diameter of $26 \pm 4.6 \mu \mathrm{m}$ ), polygonal NADPH-dreactive cells of the LDT are shown in Figure $8 D$. Cholinergic LDT-PPT neurons are also NADPH-d-reactive (Vincent et al., 1986), whereas NADPH-d-reactive cells in the Cun nucleus were not found to be cholinergic. Examples of different regions in a section treated to examine both ChAT and NADPH-d are shown in Figure $8 E-G$. With this technique, the cytoplasmic blue product of the NADPH-d NBT reaction mixes with the cytoplasmic brown reaction product of ChAT immunohistochemistry. It can be observed in Figure $8 E$ that the Cun NADPH-d positive cells were pale blue but ChAT-negative. In contrast, the majority of ChAT-positive cells within the LDT-PPT displayed NADPH-d activity. The arrowhead in Figure $8 F$ points to a cholinergic cell that does not display signs of NADPH-d activity, whereas the arrow points to a clearly double-labeled cell. All six cells illustrated in Figure $8 G$ appeared as double-labeled cells when directly examined under the microscope. The arrowheads point to three of these cells, which, in the photomicrograph, are clearly visualized as NADPH-d-reactive and ChAT-positive.

Immunohistochemistry demonstrated that NADPH-d-containing neurons also contained NOS. An example of a fusiform Fos ${ }^{+}$ Cun neuron labeled by the NOS antibody is shown in Figure $8 H$. For comparison, a LDT neuron positive for NOS immunostaining is presented in Figure 8I. It is known that neurons that contain both NOS and NADPH-d are able to produce NO; therefore, we refer to those neurons that exhibit this particular kind of immunoreactive profile as nitrergic neurons (Hope et al., 1991).

\section{DISCUSSION}

We suggest that the robust Fos immunoreactivity found in the present study in Cun neurons during AS-carbachol reflects neuronal excitation related to this state. The Cun nucleus itself has not been implicated before in AS processes, although in its immediate vicinity, in the lateral PPT, the presence of PGO on neurons has been described previously (Paré et al., 1990). Our findings indicate the need to examine the role of the Cun nucleus in AS and related physiological processes.

\section{Methodological considerations}

Using the variables that were measured, the state induced by carbachol is indistinguishable from normal AS, but it is not the natural state (Morales et al., 1987; López-Rodríguez et al., 1995; Kohlmeier et al., 1996; Xi et al., 1997; Maloney et al., 1999). AS-carbachol is of long duration, and it is therefore possible that some of the neurons that express $c$-fos do so because of an increase in their metabolism rather than because of an increase in their firing alone. However, the pharmacologically induced AS state was used to allow enough time for the Fos protein to accumulate to detectable levels (Yamuy et al., 1993; Shiromani et al., 1995). On this issue, as well as on that of the potential caveats related to the use of $c$-fos expression, we refer the reader to previous works (Dragunow and Faull, 1989; Herrera and Robertson, 1996; Chaudhuri, 1997; Maloney et al., 1999; Morales et al., 1999). It is unlikely that $c$-fos expression reflects neuronal inhibition, because it depends mostly on an increase in intracellular $\mathrm{Ca}^{2+}$ concentration that occurs during certain kinds of excitatory synaptic transmission. In addition, cells such as motoneurons, which are subjected to sustained postsynaptic inhibition during 


\section{GABA, $c$-fos}
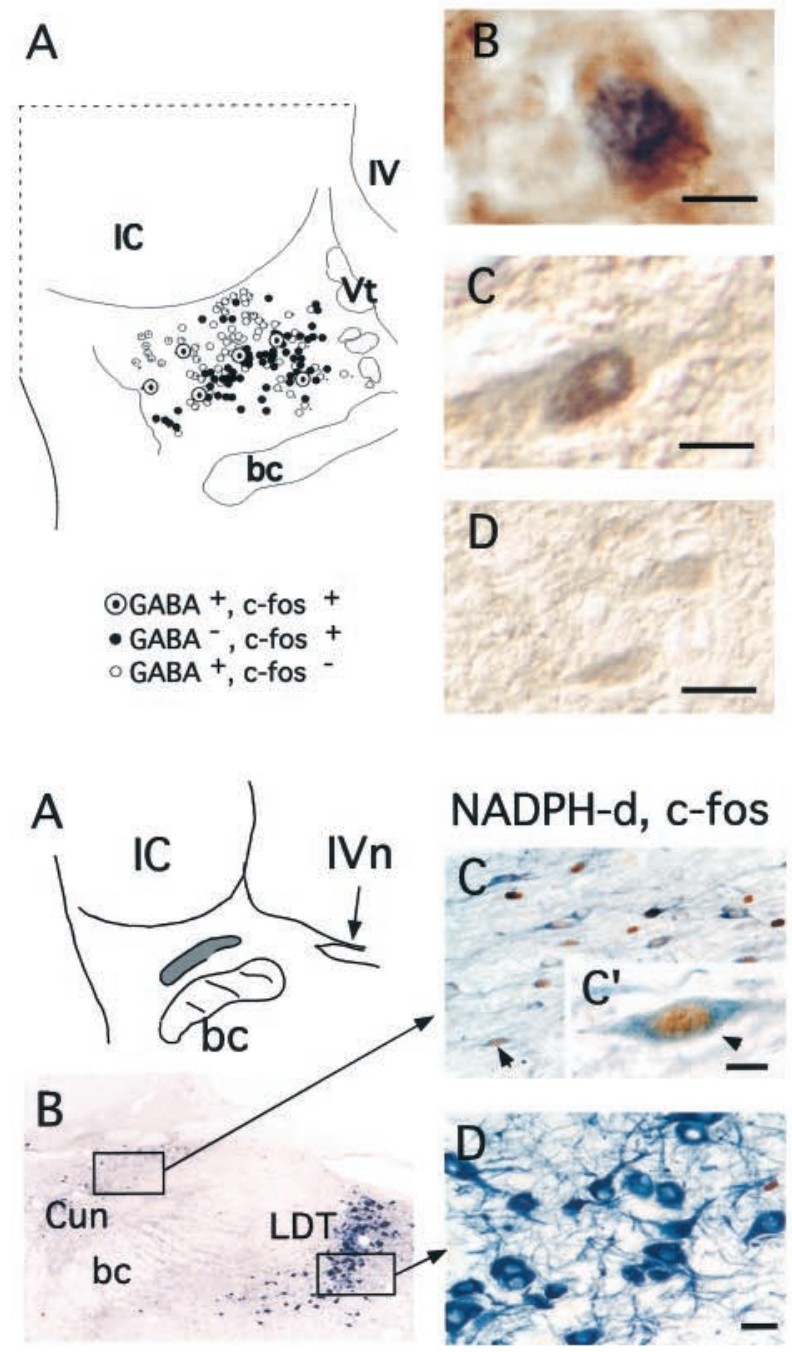

\section{NADPH-d, ChAT}
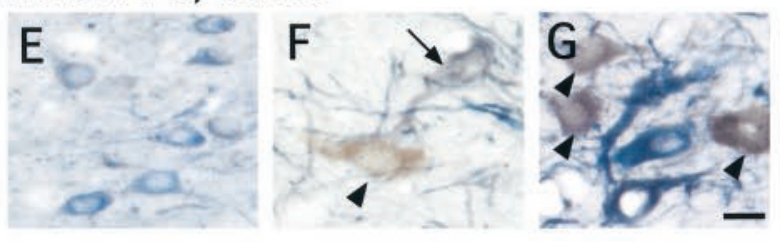

\section{NOS, c-fos}
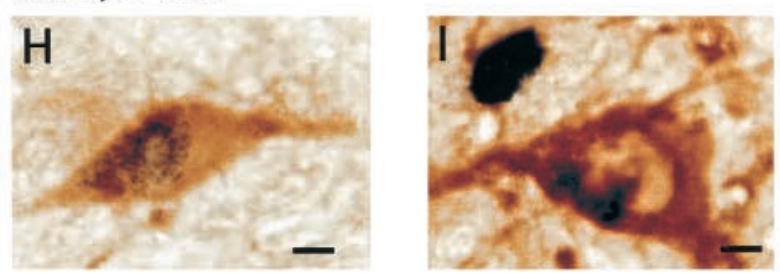

\section{NADPH-d, $c$-fos}
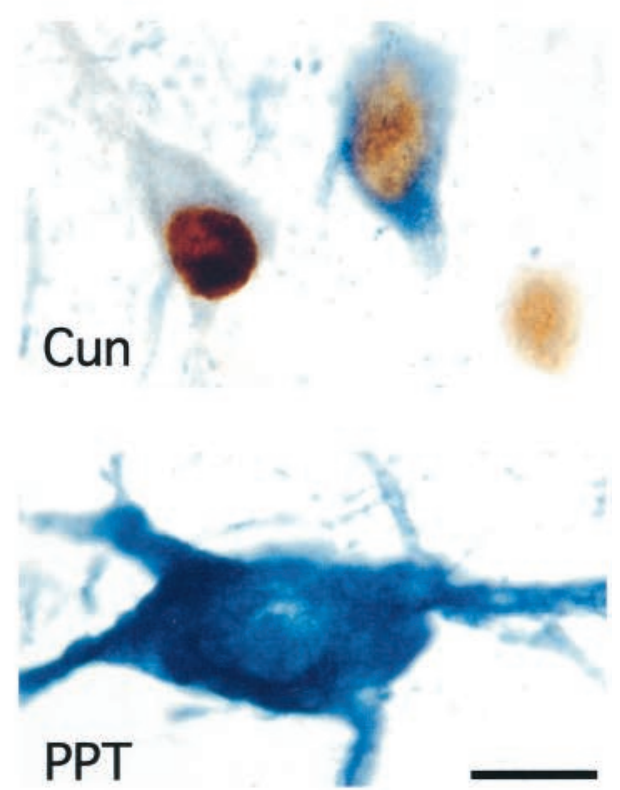

Figure 6. Top Left, GABAergic neurons in the Cun nucleus. $A$ is a diagram of one section immunostained for both GABA and Fos. Open circles represent $\mathrm{GABA}^{+}$, Fos ${ }^{-}$neurons; filled circles represent $\mathrm{Fos}^{+}, \mathrm{GABA}^{-}$neurons; and large circles with a dot represent doublelabeled $\mathrm{GABA}^{+}, \mathrm{Fos}^{+}$neurons. The histological sections were obtained from an experimental cat and processed for both GABA and Fos immunostaining in $B$ and for GAD and Fos immunostaining in $C$ and $D . B$ and $C$ are examples of putative GABAergic neurons that express $c$-fos. In $D$ are examples of $\mathrm{GAD}^{+}$neurons that do not express $c$-fos. Scale bars, $25 \mu \mathrm{m}$. $b c$, Brachium conjunctivum; $I V$, fourth ventricle; $V t$, mesencephalic tract.

Figure 7. Top Right, Differences between the NADPH-d-reactive neurons in the Cun nucleus and those within the PPT. The histological section from which these photomicrographs were taken was obtained from an experimental cat, processed for Fos protein immunostaining and treated to reveal NADPH-d activity. The top photomicrograph is from the Cun nucleus, the bottom one is from the PPT. Note the differences in size, shape, and intensity of the cytoplasmic stain between both types of cells. The Cun cells are Fos ${ }^{+}$. Scale bar, $15 \mu \mathrm{m}$.

Figure 8. Bottom, c-fos expression in a subpopulation of neurons of the nucleus Cun displaying NADPH-d activity. $A$ illustrates the location of these neurons (shaded area) in a coronal section at the level of the decussation of the fibers of the trochlear nerve ( $I V n$; arrow). $B$, Photomicrograph of a section treated to reveal neurons displaying NADPH-d activity. This section was also processed for Fos immunostaining and counterstained. $C$ is an expanded view of this region of the Cun nucleus. Note the presence of numerous $c$-fos-expressing NADPH-d ${ }^{+}$neurons. The $C^{\prime}$ inset shows a neuron of this region (arrowhead) at greater magnification. $D$ is an expanded view of the LDT nucleus containing typical NADPH-d ${ }^{+}$cholinergic neurons. $E-G$, These were taken from sections that were processed for ChAT immunostaining and treated to determine those neurons displaying NADPH-d activity. $E$ is a photomicrograph of a subpopulation of Cun nucleus neurons; these neurons were pale blue and did not exhibit ChAT immunostaining. In $F$ are two PPT neurons; the arrowhead points to a brown neuron that exhibits pure ChAT immunostaining and not NADPH-d activity. The arrow points to a bluebrown cell that displays both ChAT immunostaining and NADPH-d activity. $G$ corresponds to the region of the LDT; the arrowheads point to clearly double-labeled neurons. $H, I$, These photomicrographs were taken from the same section, which was processed for both Fos and NOS immunostaining. In $H$, a Cun neuron that expresses $c$-fos and displays NOS reactivity is shown. $I$ shows an NOS-immunoreactive, Fos ${ }^{-}$LDT neuron. Scale bars: $C^{\prime}, 20 \mu \mathrm{m} ; D$, $40 \mu \mathrm{m}$ (also applies to $C$ ); $E-G, 40 \mu \mathrm{m} ; H, I, 5 \mu \mathrm{m}$. $b c$, Brachium conjunctivum. 
AS, do not express $c$-fos during this state (Yamuy et al., 1993). Therefore, our interpretation of the data is based on the assumption that $c$-fos expression reflects neuronal activation.

\section{Neurotransmitter phenotype}

Cuneiform neurons are not a homogeneous population of cells. We observed three different types of cells in this nucleus. There was a population of GABAergic cells, most of which did not express $c$-fos during AS-carbachol, and therefore do not appear to be specifically active during the state. Two different populations of neurons did express $c$-fos. In one of these Fos ${ }^{+}$populations, we were unable to identify a neurotransmitter. The other Fos ${ }^{+}$ population consisted of nitrergic neurons. Of equivalent importance to these positive results is the absence of cholinergic, catecholaminergic, glycinergic, or serotonergic neurons in this structure. Several peptides have been shown to exist in the Cun nucleus (e.g., substance P, enkephalins, and corticotropin releasing factor) (Beitz, 1982; Sakanaka et al., 1987). Because peptides are usually colocalized with classical neurotransmitters, it is possible that cells expressing $c$-fos are also peptidergic.

The nature of the neurotransmitter in one of the populations of $\mathrm{Fos}^{+}$Cun neurons remains unknown. Although we did not detect glutamate-like reactivity in their cell bodies, there is evidence of excitatory amino acid-containing projections from this nucleus that could be glutamatergic (Beitz, 1989; Beart et al., 1990; Richter and Behbehani, 1991). A lack of sensitivity of our immunocytochemical technique is not likely to be the reason why glutamate immunoreactivity was not detected in Cun cells because glutamate-like reactivity was observed in known glutamatergic cells in the inferior colliculus and in the Mes-V nucleus in the same and adjacent sections (Fig. 5). Results from immunocytochemistry examining excitatory amino acids are difficult to interpret, particularly when one is confronted by the lack of staining in cell somas (Beitz, 1990; Storm-Mathisen and Ottersen, 1990). Glutamatergic neurons may exhibit lower levels of glutamate-like immunoreactivity in their cell bodies than in their axon terminals (Storm-Mathisen and Ottersen, 1990; Quaglino et al., 1999). Therefore, it is possible that Cun cells use glutamate as their neurotransmitter but that their perikarya do not display significant levels of immunoreactivity. Alternatively, aspartate rather than glutamate may be used by Cun cells as a neurotransmitter (McGeer et al., 1987; Robinson and Coyle, 1987).

Nitrergic Cun neurons should not be confused with the cholinergic cells of the LDT-PPT that also contain NOS and display NADPH-d activity (Vincent et al., 1986) for the following reasons. Cun neurons did not show immunoreactivity for ChAT, they were smaller than cholinergic cells in the LDT-PPT (14 \pm 0.24 vs $26 \pm 0.58 \mu \mathrm{m}$, respectively; $p<0.0001$ ), and their morphology was different (Figs. 7, 8). The fact that these Cun neurons are nitrergic, but not cholinergic, raises the question as to the conventional neurotransmitter that they use.

Fifty percent of the nitrergic Cun neurons expressed $c$-fos during AS-carbachol. To the best of our knowledge, nothing is known about their connections and functions. However, based on our present findings, it would be expected that their cell bodies and their axon terminals produce NO during AS and that this molecule modulates the activity of the targets for Cun neurons. In this regard, the Cun nucleus is the origin of ascending and descending pathways. Its ascending fibers project to the hypothalamus, to the central amygdala, and to several thalamic nuclei (parafascicular, centromedian, and centrolateral) (Edwards and de Olmos, 1976; Zemlan and Behbehani, 1988; Korte et al.,
1992). In the lateral geniculate nucleus (LGN), NO is produced in greater amounts during AS (Williams et al., 1997). LGN neurons are modulated by NO (Pape and Mager, 1992), likely produced in the axon terminals from LDT-PPT neurons. By analogy, we suggest that the NO that would be produced by cuneiform axon terminals modulates the activity of other thalamic nuclei during AS. Descending Cun fibers mainly innervate the nucleus raphe magnus (RM) and the giganto- and magno-cellularis nuclei (NRGC and NMC, respectively) (Edwards, 1975; Zemlan and Behbehani, 1988; Bernard et al., 1989). Within RM, NRGC, and NMC nuclei, we have found larger numbers of $\mathrm{Fos}^{+}$neurons during AS-carbachol than during wakefulness and have suggested that they are activated during AS-carbachol (Yamuy et al., 1993, 1995; Morales et al., 1999). Maloney et al. (1999), in turn, have reported an increase in the number of GABAergic neurons within the RM during the recovery of AS in rats. Cun Fos ${ }^{+}$ neurons could be one of the sources of the activation of cells within these nuclei, and NO may modulate the behavior of their neurons.

\section{Relevance to sleep physiology}

Convincing evidence has been presented in previous studies indicating that NO produced by LDT-PPT cholinergic neurons is involved in the modulation of sleep and waking states, as well as in the release of acetylcholine in the rostral pontine tegmentum (Datta et al., 1997; Leonard and Lydic, 1997; Hars, 1999). In the present study, we found that a subpopulation of Cun neurons could also be an important source of NO produced during AS. However, it is not possible to assign a single physiological role to Cun neurons during AS because of the heterogeneity of functions in which the Cun nucleus has been implicated. In this regard, this nucleus is part of the mesencephalic locomotor region (Grillner, 1981; Garcia-Rill and Skinner, 1988; Mori et al., 1989). Interestingly, animals under picrotoxin-induced locomotor activity display an increase in c-fos-expressing cells in this nucleus (Brudzynski and Wang, 1996). However, compared with the number of Fos $^{+}$cells observed in the present work, the number of $c$-fosexpressing cells during induced locomotion is two orders of magnitude lower. In other studies, the Cun nucleus has been considered important in the integration of cardiovascular-autonomic functions (Korte et al., 1992; Lam et al., 1996). The Cun nucleus may be also involved in defensive or stress-related behaviors (Korte et al., 1992), a condition during which there is also evidence of increased $c$-fos expression (Kollack-Walker et al., 1997).

The activation of the Cun neurons during AS may be involved in three processes that take place during this state. The first, that was discussed above, relates to the possible regulation of thalamic neurons during AS. The second is antinociception. It was only recently that this process was demonstrated to occur after the administration of cholinomimetic substances in the pons and during cholinergically induced AS (Kshatri et al., 1998). The Cun nucleus is a component of the descending anti-nociceptive system (Dostrovsky et al., 1983; Katayama et al., 1984; Zemlan and Behbehani, 1988; Richter and Behbehani, 1991). The RM, which is also part of this system and exhibits strong $c$-fos expression during AS-carbachol (see above), is heavily innervated by Cun fibers (Leung and Mason, 1999; Mason, 1999). It is likely that cholinergic activation of the rostral pontine tegmentum during AS results in antinociception caused by the subsequent activation of the above mentioned structures.

The third process that may be regulated by Cun activation 
during AS is motor inhibition. Electrical stimulation of the Cun nucleus elicits suppression of muscular tone and somatic reflexes (Mileikovsky et al., 1989, 1990; Mileikovsky and Nozdrachev, 1997). These effects, we suggest, may be mediated by premotor inhibitory neurons within the ventromedial medulla (Morales et al., 1999). The ventromedial medulla is one of the principal targets of Cun fibers. It is possible that Cun terminals modulate the activity of premotor neurons during AS through a synergistic mechanism that involves excitatory neurotransmitters and modulatory nitrergic actions.

\section{REFERENCES}

Appell PP, Behan M (1990) Sources of subcortical GABAergic projections to the superior colliculus in the cat. J Comp Neurol 302:143-158. Baghdoyan HA, Rodrigo-Angulo ML, McCarley RW, Hobson JA (1987) A neuroanatomical gradient in the pontine tegmentum for the cholinoceptive induction of desynchronized sleep signs. Brain Res 414:245-261.

Baghdoyan HA, Lydic R, Callaway CW, Hobson JA (1989) The carbachol-induced enhancement of desynchronized sleep is dose dependent and antagonized by centrally administered atropine. Neuropsychopharmacology 2:67-69.

Beart PM, Summers RJ, Stephenson JA, Cook CJ, Christie MJ (1990) Excitatory aminoacid projections to the periaqueductal gray in the rat: a retrograde transport study utilizing $\mathrm{D}\left[{ }^{3} \mathrm{H}\right]$ aspartate and $\left[{ }^{3} \mathrm{H}\right] \mathrm{GABA}$. Neuroscience 34:163-176.

Beitz AJ (1982) The nuclei of origin of brain stem enkephalin and substance $\mathrm{P}$ projections to the rodent nucleus raphe magnus. Neuroscience 7:2753-2768.

Beitz AJ (1989) Possible origin of glutamatergic projections to the midbrain periaqueductal gray and deep layers of the superior colliculus of the rat. Brain Res Bull 23:25-35.

Beitz AJ (1990) Relationship of glutamate and aspartate to the periaqueductal gray-raphe magnus projection: analysis using immunocytochemistry and microdialysis. J Histochem Cytochem 38:1755-1765.

Berman AL (1968) The brainstem of the cat. A cytoarchitectonic atlas with stereotaxic coordinates. Madison, WI: University of Wisconsin.

Bernard JF, Peschansky M, Besson JM (1989) Afferents and efferents of the cuneiform nucleus: an anatomical study with reference to pain transmission. Brain Res 490:181-185.

Brudzynski SM, Wang D (1996) C-Fos immunohistochemical localization of neurons in the mesencephalic locomotor region in the rat brain. Neuroscience 75:793-803.

Capece ML, Baghdoyan HL, Lydic R (1998) Carbachol stimulates (35S) guanylyl $5^{\prime}$-( $\gamma$-thio)-triphosphate binder in REM sleep-related brain stem nuclei of the rat. J Neurosci 18:3779-3785.

Chaudhuri A (1997) Neural activity mapping with inducible transcription factors. NeuroReport 8:v-ix.

Datta S, Patterson EH, Siwek DF (1997) Endogenous and exogenous nitric oxide in the pedunculopontine tegmentum induces sleep. Synapse 27:69-78.

Dostrovsky JO, Shah Y, Gray BG (1983) Descending inhibitory influences from periaqueductal gray, nucleus raphe magnus, and adjacent reticular formation. II. Effects on medullary dorsal horn nociceptive and nonnociceptive neurons. J Neurophysiol 49:948-960.

Dragunow M, Faull R (1989) The use of $c$-fos as a metabolic marker in neuronal pathway tracing. J Neurosci Methods 29:261-265.

Edwards SB (1975) Autoradiographic studies of the projections of the midbrain reticular formation: descending projections of nucleus cuneiformis. J Comp Neurol 161:341-358.

Edwards SB, de Olmos JS (1976) Autoradiographic studies of the projections of the midbrain reticular formation: ascending projections of nucleus cuneiformis. J Comp Neurol 165:417-431.

Fulwiler CE, Saper CB (1984) Subnuclear organization of the efferent connections of the parabrachial nucleus in the rat. Brain Res Rev 7:229-259.

Garcia-Rill E, Skinner RD (1988) Modulation of rhythmic function in the posterior midbrain. Neuroscience 27:639-654.

George R, Haslett WL, Jenden DJ (1964) A cholinergic mechanism in the brainstem reticular formation: induction of paradoxical sleep. Int J Neuropharmacol 3:541-552.

Gioia M, Bianchi R (1987) The cytoarchitecture of the nucleus cuneiformis. A Nissl and Golgi study. J Anat 155:165-176.
Grillner S (1981) Control of locomotion in bipeds, tetrapeds and fish. In: Handbook of physiology. The nervous system, Vol 2, Motor control (Brookhart JM, Mountcastle VB, Brooks VB, eds), pp 1179-1236. Bethesda, MD: American Physiology Society.

Hars B (1999) Endogenous nitric oxide in the rat pons promotes sleep. Brain Res 816:209-219.

Herrera DG, Robertson HA (1996) Activation of $c$-fos in the brain. Prog Neurobiol 50:83-107.

Hobson JA (1992) Sleep and dreaming: induction and mediation of REM sleep by cholinergic mechanisms. Curr Opin Neurobiol 2:759-763.

Hope BT, Michael GJ, Knigge KM, Vincent SR (1991) Neuronal NADPH diaphorase is a nitric oxide synthase. Proc Nat Acad Sci USA 88:2811-2814.

Jones BE (1991) Paradoxical sleep and its chemical/structural substrates in the brain. Neuroscience 40:637-656.

Jones BE, Beaudet A (1987) Distribution of acetylcholine and catecholamine neurons in the cat brain stem studied by choline acetyltransferase and tyrosine hydroxilase immunohistochemistry. J Comp Neurol 261:15-32.

Katayama Y, DeWitt DS, Becker DP, Hayes RL (1984) Behavioral evidence for a cholinoceptive pontine inhibitory area: descending control of spinal motor output and sensory input. Brain Res 296:241-264.

Kohlmeier KA, López-Rodríguez F, Liu RH, Morales FR, Chase MH (1996) State dependent phenomena in cat masseter motoneurons. Brain Res 722:30-38.

Kollack-Walker S, Watson SJ, Akil H (1997) Social stress in hamsters: defeat activates specific neurocircuits within the brain. J Neurosci 17:8842-8855.

Korte SM, Jaarsma D, Luiten PG, Bohus B (1992) Mesencephalic cuneiform nucleus and its ascending and descending projections serve stress-related cardiovascular responses in the rat. J Auton Nerv Syst 41:157-176.

Kshatri AM, Baghdoyan HA, Lydic R (1998) Cholinomimetics, but not morphine, increase antinociceptive behavior from pontine reticular regions regulating rapid-eye-movement sleep. Sleep 21:677-685.

Lam W, Gundlach AL, Verberne AJ (1996) Increased nerve growth factor inducible-A gene and c-fos messenger RNA levels in the rat midbrain and hindbrain associated with the cardiovascular response to electrical stimulation of the mesencephalic cuneiform nucleus. Neuroscience 71:193-211.

Leonard TO, Lydic R (1997) Pontine nitric oxide modulates acetylcholine release, rapid eye movement sleep generation, and respiratory rate. J Neurosci 17:774-785.

Leung CG, Mason P (1999) Physiological properties of raphe magnus neurons during sleep and waking. J Neurophysiol 81:584-595.

López-Rodríguez F, Kohlmeier K, Yamuy J, Morales FR, Chase MH (1995) Muscle atonia can be induced by carbachol injections into the nucleus pontis oralis in cats anesthetized with a-chloralose. Brain Res 699:201-207.

Maloney KJ, Mainville L, Jones BE (1999) Differential c-Fos expression in cholinergic, monoaminergic, and GABAergic cell groups of the pontomesencephalic tegmentum after paradoxical sleep deprivation and recovery. J Neurosci 19:3057-3072.

Mason P (1999) Central mechanisms of pain modulation. Curr Opin Neurobiol 9:436-441.

McGeer PL, Eccles JC, McGeer EG (1987) Putative excitatory neurons: glutamate and Aspartate. In: Molecular neurobiology of the mammalian brain. Ed 2 (McGeer PL, Eccles JC, McGeer EG, eds), pp 175-196. New York: Plenum.

Merchant-Nancy H, Vázquez J, Aguilar-Roblero R, Drucker-Colin R (1992) C-fos proto-oncogene changes in relation to REM sleep duration. Brain Res 579:342-346.

Mileikovsky BY, Nozdrachev AD (1997) Electrophysiological analysis of interaction between the pons and medulla, the brainstem inhibitory areas. Fiziol Zh SSSR Im I M Sechenova 83:57-63.

Mileikovsky BY, Verevkina SV, Nozdrachev AD (1989) The participation of the inhibitory locomotor system of the brain stem in regulating pain sensitivity. Fiziol Zh SSSR Im I M Sechenova 75:1051-1056.

Mileikovsky BY, Verevkina SV, Nozdrachev AD (1990) The central neurophysiological mechanisms in the regulation of inhibition. Fiziol Zh SSSR Im I M Sechenova 76:289-294.

Morales FR, Engelhardt JK, Soja PJ, Pereda AE, Chase MH (1987) Motoneuron properties during motor inhibition produced by microin- 
jection of carbachol into the pontine reticular formation of the decerebrate cat. J Neurophysiol 57:1118-1129.

Morales FR, Sampogna S, Yamuy J, Chase MH (1999) c-Fos expression in brainstem premotor interneurons during cholinergically-induced active sleep in the cat. J Neurosci 19:9508-9518.

Mori S, Sakamoto T, Ohta Y, Takakusaki K, Matsuyama K (1989) Site-specific postural and locomotor changes evoked in awake, freely moving intact cats by stimulating the brainstem. Brain Res 505:66-74.

Mugnaini E, Oertel WH (1985) An atlas of the distribution of GABAergic neurons and terminals in the rat CNS as revealed by GAD immunohistochemistry. In: Handbook of chemical neuroanatomy, Vol 4, GABA and neuropeptides in the CNS, Pt I (Bjorklund A, Hokfelt T, eds). Amsterdam: Elsevier.

Pape HC, Mager R (1992) Nitric oxide controls oscillatory activity in thalamocortical neurons. Neuron 9:441-448.

Paré D, Curró Dossi R, Datta S, Steriade M (1990) Brainstem genesis of reserpine-induced ponto-geniculo-occipital waves: an electrophysiological and morphological investigation. Exp Brain Res 81:533-544.

Quaglino E, Giustetto M, Panzanelli P, Cantino D, Fasolo A, Sassoè Pognetto M (1999) Immunocytochemical localization of glutamate and gamma-aminobutyric acid in the accessory olfactory bulb of the rat. J Comp Neurol 408:61-72.

Richter RC, Behbehani MM (1991) Evidence for glutamic acid as a possible neurotransmitter between the mesencephalic nucleus cuneiformis and the medullary nucleus raphe magnus in the lightly anesthetized rat. Brain Res 544:279-286.

Robinson MB, Coyle JT (1987) Glutamate and related acidic excitatory neurotransmitters: from basic science to clinical application. FASEB J $1: 446-455$

Rye DB (1997) Contributions of the pedunculopontine region to normal and altered REM sleep. Sleep 20:757-788.

Rye DB, Saper CB, Lee HJ, Wainer BH (1987) Pedunculopontine tegmental nucleus of the rat: cytoarchitecture, cytochemistry and some extrapyramidal connections of the mesopontine tegmentum. J Comp Neurol. 259:483-528.

Sakai K (1988) Executive mechanisms of paradoxical sleep. Arch Ital Biol 126:239-257.

Sakanaka M, Shibasaki T, Lederis K (1987) Corticotropin releasing factor-like immunoreactivity in the rat brain as revealed by a modified cobalt-glucose oxidase-diaminobenzidine method. J Comp Neurol 260:256-298.

Shiromani JP, Kilduff TS, Bloom FE, McCarley RW (1992) Cholinergically induced REM sleep triggers Fos-like immunoreactivity in dorso- lateral pontine regions associated with REM sleep. Brain Res 580:351-357.

Shiromani JP, Malik M, Winston S, McCarley RW (1995) Time course of $f o s$-like immunoreactivity associated with cholinergic induced REM sleep. J Neurosci 15:3500-3508.

Shuman SL, Capece ML Baghdoyan HL Lydic R (1995) Pertussis toxinsensitive $G$ proteins mediate carbachol-induced REM sleep and respiratory depression. Am J Physiol 269:R308-R317.

Siegel JM (1994) Brainstem mechanisms generating REM sleep. In: Principles and practice of sleep medicine (Kryger MH, Roth T, Dement WC, eds), pp125-144. Philadelphia: Saunders.

Steriade M, McCarley RW (1990) Brainstem control of wakefulness and sleep (Steriade M, McCarley RE, eds). New York: Plenum.

Storm-Mathisen J, Ottersen OP (1990) Immunocytochemistry of glutamate at the synaptic level. J Histochem Cytochem 38:1733-1743.

Vanni-Mercier G, Sakai K, Lin JS, Jouvet M (1989) Mapping of cholinoceptive brainstem structures responsible for the generation of paradoxical sleep in the cat. Arch Ital Biol 127:133-164.

Vincent SR, Reiner PB (1987) The immunohistochemical localization of choline acetyltransferase in the cat brain. Brain Res Bull 18:371-415.

Vincent SR, Satoh K, Armstrong DM, Panula P, Vale W, Fibiger HC (1986) Neuropeptides and NADPH-diaphorase activity in the ascending cholinergic reticular system of the rat. Neuroscience 17:167-182.

Williams JA, Vincent SR, Reiner PB (1997) Nitric oxide production in rat thalamus changes with behavioral state, local depolarization, and brainstem stimulation. J Neurosci 17:420-427.

Xi MC, Liu RH, Yamuy J, Morales FR, Chase MH (1997) Electrophysiological properties of lumbar motoneurons in the a-chloraloseanesthetized cat during carbachol-induced motor inhibition. J Neurophysiol 78:129-136.

Yamuy J, Mancillas JR, Morales FR, Chase MH (1993) c-Fos expression in the pons and medulla of the cat during carbachol-induced active sleep. J Neurosci 13:2703-2718.

Yamuy J, Sampogna S, López-Rodríguez F, Luppi PH, Morales FR, Chase MH (1995) Fos and serotonin immunoreactivity in the raphe nuclei of the cat during carbachol-induced active sleep: a doublelabeling study. Neuroscience 67:211-223.

Yamuy J, Sampogna S, Morales FR, Chase MH (1998) c-Fos expression in mesopontine noradrenergic and cholinergic neurons of the cat during carbachol-induced active sleep: a double-labeling study. Sleep Res On-line 1:28-40.

Zemlan FP, Behbehani MM (1988) Nucleus cuneiformis and pain modulation: anatomy and behavioral pharmacology. Brain Res 453:89-102. 